\title{
Practices and Attitudes toward Returning Genomic Research Results to Low-Resource Research Participants
}

\author{
Megan B. Raymond a, b Kayla E. Cooper ${ }^{a}$ Lisa S. Parker ${ }^{c}$ Vence L. Bonham ${ }^{a}$ \\ aSocial and Behavioral Research Branch, National Human Genome Research, National Institutes of Health,

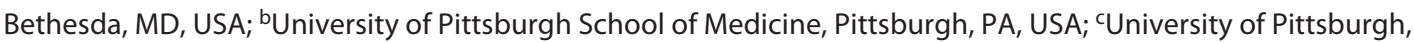 \\ Center for Bioethics \& Health Law, Human Genetics, Graduate School of Public Health, Pittsburgh, PA, USA
}

\section{Keywords}

Genomics · Research ethics · Underserved communities ·

Health equity $\cdot$ Return of results

\begin{abstract}
Introduction: Many research programs are challenged to accommodate low-resource research participants' (LRRP) ancillary care needs when returning genomic research results. We define LRRP as those who are low income, uninsured, underinsured, or facing barriers to act upon the results returned. This study evaluates current policies and practices surrounding return of results (RoR) to LRRP, as well as the attitudes of investigators toward providing ancillary care to LRRP. Methods: A semi-structured interview study was conducted with representatives of 35 genomic research programs nationwide. Eligible programs were returning, or planning to return, medically actionable genomic results to participants. Results: Three content categories emerged from this study, including: (1) RoR structures, (2) barriers to RoR to LRRP, and (3) solutions to meet community and LRRP needs. Three major structures of RoR emerged: (1) RoR Embedded in Clinical Care, (2) RoR Independent of Clinical Care,
\end{abstract}

and (3) Reliance on Clinical Partnerships to Facilitate RoR. Inadequacy of program resources to address the needs of LRRP was commonly considered a significant obstacle. The attitudes and views of informants regarding responsibility to provide ancillary care for LRRP receiving genomic results were highly varied. Some informants believed that genomic sequencing and testing was not a priority for LRRP because of other pressing issues in their lives, such as housing and food insecurity. Research programs differ regarding whether clinical and social support for LRRP is considered within the purview of the research team. Some programs instituted accommodations for LRRP, including social work referral and insurance enrollment assistance. Conclusion: Support to access downstream treatment is not readily available for LRRP in many genomic research programs. Development of best practices and policies for managing RoR to LRRP is needed.

(C) 2021 The Author(s)

Published by S. Karger AG, Basel

Megan B. Raymond and Kayla E. Cooper are co-first authors, and these authors contributed equally to the manuscript. karger@karger.com www.karger.com/phg

Karger $\stackrel{\text { ' }}{5}$

BOPEN ACCESS
(C) 2021 The Author(s)

Published by S. Karger AG, Basel

This is an Open Access article licensed under the Creative Commons Attribution-NonCommercial-4.0 International License (CC BY-NC) (http://www.karger.com/Services/OpenAccessLicense), applicable to the online version of the article only. Usage and distribution for commercial purposes requires written permission.
Correspondence to:

Vence L. Bonham, bonhamv@mail.nih.gov 


\section{Introduction}

The significant reduction in the cost of next-generation sequencing has led to an increase in the number of research participants who have their genomes sequenced [1]. There is little known about the views of investigators in genomic and precision medicine research programs regarding the return of genomic results to low-resource research participants (LRRP). We define LRRP as individuals who are low income, uninsured, underinsured, living in a medically underserved area, or facing significant barriers to act upon their genomic results in the USA. Researchers are obligated to consider their ethical responsibilities when returning actionable genomic research results to study participants, "in a way that does not discriminate on the basis of economic ability" [2]. Some researchers contend that low genetic literacy and the potential for psychological distress associated with return of results (RoR) require tailoring the RoR process for these study participants $[3,4]$.

In response to the debate regarding RoR, the National Academies of Sciences, Engineering, and Medicine (NAS$\mathrm{EM}$ ) in the USA convened a consensus panel to consider principles and practices for RoR and concluded that researchers are "ethically obligated to return urgent, medically actionable research results to their participants" [2]. The NASEM report emphasizes that investigators should return results in a way that "accommodates the full spectrum of community needs and preferences, regardless of participant's social or economic status" [2]. The NASEM report also highlights that "the challenges encountered in engaging disenfranchised groups and any concerns related to a lack of resources (e.g., access to follow-up health care) are not justifiable reasons for denying potential benefits from the return of results" $[2,5]$.

The ethics of RoR to diverse populations is salient in light of the scientific community's commitment to increase the diversity of research participants in genomic studies to foster understanding of benign and pathogenic gene variants in different ancestral populations [6-10]. Though it is important not to equate populations of nonEuropean ancestry with LRRP, achieving recruitment goals of ancestrally diverse populations, for genomic sequencing studies in the USA, will involve recruiting LRRP [11]. Engagement of underrepresented populations in genomic research has been studied in various settings [1214]. Several studies have examined how to return results to various demographic groups, including minors, adults who lack decision-making capacity, and relatives of deceased persons [15-20]. Very few studies have examined
RoR in low-resource clinical environments such as federally qualified health centers [21]. There is a lack of scientific community consensus concerning whether ancillary care or social support should be provided to LRRP following RoR.

The ethical principles developed by Beauchamp and Childress [22] have been used to maintain that there is a duty to rescue a person when the action will likely succeed in preventing harm. Scholars have written about the "duty to rescue" as a moral obligation relevant for the return of secondary findings in genomic studies [23]. Furthermore, it has been argued that beneficence includes an obligation to implement "reasonable measures to help individuals with actionable genetic variants that have clinical significance" [24].

Researchers in the USA are facing the ethical challenges of an increasingly blurring research-clinical divide [25], which has resulted in what has been described as "clinical-esque" obligations for genomic researchers [26]. The American Society of Human Genetics examined the responsibility to recontact research participants after reinterpretation of genetic and genomic research results. The 12 recommendations reported explicitly do not address whether there is a responsibility to provide ancillary care, stating that clinical contexts are outside the scope of the position statement. However, they do reference the necessary limits of ancillary care:"It is necessary for researchers to carefully consider how to pursue scientific knowledge by using an approach that confers the best possible balance of risks and benefits to participants while still generating the benefits of high-quality research. In other words, any responsibility that researchers have to provide benefits to their research participants (also known as an ancillary-care responsibility) is necessarily a limited responsibility" [27].

We examine the ethical framework of "ancillary care" for LRRP and contend that, in certain circumstances, researchers have a responsibility to provide resources for medical needs that go beyond the regulatory requirements of safety or rectifying injuries [28-31]. We also examine the concept of "social care" responsibilities of researchers. While not directly a component of the definition of ancillary care first proposed by Richardson and Belsky [28], social care is also an important consideration. Social care is described as the services that address healthrelated social risk factors and social needs (e.g., transportation, childcare, and social services) [32].

The concept of ancillary care came to prominence through global health research, primarily in studies in low- and middle-income countries (LMIC) investigating 
HIV/AIDS interventions [33-35]. Since then, it has been extended to group-focused public health research in developing countries as well [36]. Ethicists have discussed concerns regarding the RoR in LMIC due to the "actionability problem" - the inability for research participants to access the same level of health care, based on results, that is available in high-income countries such as the USA. There continues to be debate regarding the return of secondary genomic findings to research participants in low-resource settings in LMIC countries [35].

However, the debate of ancillary care is not limited to low-income countries: "research participants in high income countries who are uninsured or otherwise lack access to healthcare encounter limitations similar to those faced by participants in LMIC" [37]. In a literature review study of the ethics of uninsured participants accessing health care through research studies, Cho et al. [37] examine the ancillary care debate in high-income countries. They found that some researchers assert that providing ancillary care could increase participants' misunderstanding of the purpose of research, expand occurrence of therapeutic misconception, and may serve as a potential disincentive for researchers to recruit from low-income communities [38]. Other scholars have argued that ancillary care is a form of coercion of low-income study participants. "The quid pro quo arrangement of offering medical care in exchange for study participation would be no different to the payment of research subjects (in fact, it could mean greater financial incentives for poorer subjects)" [39].

Institutional review board (IRB) professionals and researchers are faced with determining what is feasible support when returning results in a research setting $[40,41]$. While specific guidance was provided by NASEM for many aspects of the RoR process, explicit guidance on how researchers should accommodate and support research participants from low-resource and disadvantaged backgrounds in the USA was not addressed. We examine how researchers are approaching the decision to provide social and ancillary care following the return of genomic results to LRRP.

\section{Materials and Methods}

\section{Study Design}

This qualitative study was designed to evaluate the management of LRRP's medical and social ancillary needs following RoR within individual genomic research programs. A semi-structured interview guide was developed, piloted with 2 content experts in the field, and then employed in semi-structured telephone inter- views with representatives of genomic research programs. Prior to beginning the interviews, an oral script was used to describe the aims of the study, procedures to maximize the interviewees' privacy, and maintain the confidentiality of the data. Study interviewees provided verbal consent to participate in the study. This study and the verbal consent process were reviewed and approved by expedited review by the Institutional Review Board at the National Human Genome Research Institute (14-HG-0125) on May 24, 2018.

\section{Recruitment and Participants}

Genomic research programs were identified through an electronic search of NIH RePORTER and existing research programs for NIH-funded genomic research consortia, large-scale genomic research programs, and biobanks. Programs were included if they were currently sequencing or planning to sequence the genomes of research participants and either currently returning individual genomic results or planning to return results. Study interviewees included principal investigators, co-investigators, genetic counselors, and program managers representing each program (Table 1). The interviews were conducted between June and September 2018.

We organized the genomic programs into 5 categories: small $(<25,000$ participants), medium (25,001-200,000 participants), large (>200,001 participants), diseased-focused (programs with an interest in a particular disease), and disease-agnostic (programs without a focus on 1 disease). We also identified programs that were interested in recruiting a particular demographic of participants, as opposed to general population-focused studies. Each telephone interview with program representatives, conducted by M.B.R., lasted between $45 \mathrm{~min}$ and an hour.

\section{Data Analysis}

Interviews were audio-recorded during each session and transcribed verbatim. An initial coding structure was created by M.B.R. and K.E.C. based on the interview question guide and approved by V.L.B. The preliminary coding structure was modified to incorporate additional themes identified. Each code was defined. The interview transcripts were independently coded by M.B.R. and K.E.C. using NVivo 11 software to facilitate the thematic analysis. Transcript data were categorized using conventional content analysis, described by Shannon and Hsieh [42]. All codes were compared, and discrepancies were discussed through phone-call conversations between M.B.R. and K.E.C. Discrepancies in coding were solved through contextual review of each quote and the code definitions. The transcript coding had a percentage agreement score of $>90 \%$. Descriptive analysis was utilized for demographic data, RoR procedures, and comparisons of genomic research program resources for LRRP.

\section{Results}

Representatives from 38 genomic research programs, including consortia and biobanks, that met study eligibility requirements were contacted with an $81.5 \%$ response rate. Thirty-seven representatives from 35 different genomic research programs participated in this study with current genomic program enrollment ranging from 200 
Table 1. Demographics of genomic research programs

\begin{tabular}{|c|c|}
\hline Characteristic & $N=35(\%)$ \\
\hline \multicolumn{2}{|l|}{ Informant position } \\
\hline PI & $20(57)$ \\
\hline Co-PI & $8(23)$ \\
\hline Genetic counselor & $4(11)$ \\
\hline Project manager & $3(9)$ \\
\hline \multicolumn{2}{|l|}{ Geographical region } \\
\hline Northeast & $9(26)$ \\
\hline Southeast & $11(31)$ \\
\hline Midwest & $3(9)$ \\
\hline Southwest & $3(9)$ \\
\hline West & $6(16)$ \\
\hline Multi-region & $3(9)$ \\
\hline \multicolumn{2}{|c|}{ Number of research participants per program } \\
\hline$<500$ & $8(22)$ \\
\hline $500-4,999$ & $17(49)$ \\
\hline $5,000-49,999$ & $4(11)$ \\
\hline $50,000-199,999$ & $3(9)$ \\
\hline$>200,000$ & $3(9)$ \\
\hline \multicolumn{2}{|c|}{ Demographic-specific programs } \\
\hline Yes 1 & $26(74)$ \\
\hline No & $9(26)$ \\
\hline \multicolumn{2}{|l|}{ Disease-focused programs } \\
\hline Yes & $13(37)$ \\
\hline No & $22(63)$ \\
\hline \multicolumn{2}{|l|}{ Biobanks } \\
\hline Yes & $5(14)$ \\
\hline No & $30(86)$ \\
\hline \multicolumn{2}{|l|}{ Discloser of genomic results ${ }^{a}$} \\
\hline Participants' provider & $23(67)$ \\
\hline Genetic counselor & $19(55)$ \\
\hline Clinical geneticist & $7(21)$ \\
\hline Return of results service & $3(9)$ \\
\hline
\end{tabular}

${ }^{a}$ Genomic research programs may use more than 1 discloser to return a result.

to over 200,000 participants (see Table 1). Four genomic research programs had $>1$ informant, and 2 informants were interviewed about $>1$ genomic research program. We report the attitudes of informants in this study toward RoR, ancillary care, and social care for LRRP. Three broad content categories emerged from the study, including (1) RoR structures, (2) barriers for LRRP following RoR, and (3) solutions to meet community and LRRP needs.

\section{RoR Structures}

There was a high degree of variation among programs regarding the RoR process. Although there were differing approaches to RoR, we identified 3 general genomic program models: (1) RoR Embedded in Clinical Care, (2) RoR Independent of Clinical Care, and (3) Reliance on
Clinical Partnerships to Facilitate RoR, which are shown in Table 2. We also assessed which aspects of the RoR process were viewed as either research or clinical team responsibilities.

RoR Embedded in Clinical Care

Several research programs were highly integrated with a health-care system and considered RoR to be a research activity that is integrated into clinical care (shown in Table 2). Researchers would sequence participants, but clinicians returned results and facilitated any follow-up care required."We sort of integrated the research arm of the study with the clinical flow of the patient. We were working to find answers for the patient. It wasn't purely for a research basis. These were patients who were looking for answers. So, the results were returned to them as part of the clinical flow." (Informant 29, Small Disease-Focused Program).

\section{RoR Independent of Clinical Care}

Some research programs were more autonomous and took full responsibility for returning results to participants (shown in Table 2). "What we are doing currently is having the genetic counselors set aside certain days that they're working on research versus in-clinic. I don't think that people consider the sort of research to be part of clinical care yet". (Informant 23, Small Disease-Focused Program).

The self-sufficient genomic programs utilized their own research program resources to support participants through the recruitment, informed consent, and RoR processes. This included providing the funding for aids such as transportation assistance and genetic counseling services for participants and their family members, as well as covering the costs of Clinical Laboratory Improvement Amendment (CLIA) approved laboratory testing. They also coordinated or referred study participants to subsequent medical care based on the genomic results returned.

\section{Reliance on Clinical Partnerships to Facilitate the}

\section{RoR Process}

The third approach is a hybrid of the previous 2 research program models. Genomic sequencing was considered a research activity and separate from clinical care (shown in Table 2). However, these genomic programs relied on clinical colleagues to return results and provide support services to research participants. "We are really trying to allow clinical care to happen as it normally would the clinical team - the geneticists - will be return- 
Table 2. Genomic research programs' RoR structures

\begin{tabular}{ll}
\hline Themes & Quotes \\
\hline $\begin{array}{l}\text { RoR Embedded in } \\
\text { Clinical Care }\end{array}$ & $\begin{array}{l}\text { "The study is a part of standard clinical care, so we're integrating into standard clinical care for this study } \\
\text { rather than having this separated out as a research project." (Informant 27, Small, Disease-Focused } \\
\text { Program) }\end{array}$ \\
$\begin{array}{ll}\text { "Because while we are the research protocol, we are very much embedded with the clinicians, and it's a } \\
\text { partnership." (Informant 05, Small Disease-Agnostic Program) }\end{array}$ \\
$\begin{array}{l}\text { "We are needing to integrate into normal clinical practice without being disruptive to normal clinical } \\
\text { practice. I think there's many challenges with that, including the fact that you don't want to be detaining } \\
\text { Clinical Care } \\
\text { patients or parents with sick kids for too long in the day. And if they're going in for example, for a cardiac } \\
\text { workup, they're going to be there for half a day anyway, and then you just have to be cognizant of how } \\
\text { stressed they are or what else is going on in the normal course of clinical care." (Informant 27, Small } \\
\text { Disease-Focused Program) }\end{array}$ \\
\hline
\end{tabular}

Reliance on Clinical Partnerships to Facilitate RoR Process

"We're trying to strike the balance between leveraging existing resources and then building, as part of the team, the rest of it. So, where we can, we will leverage existing clinical resources." (Informant 18, Large Disease-Agnostic Program)

Research Team Responsibilities "We disclose the results, and then basically direct them back to their primary care provider to follow up Clinical Team Responsibilities on those recommended actions." (Informant 23, Small Disease-Focused Program)

"The research study is with a clinical population. Some of these patients may have received those kinds of services as part of their clinical care. But, you know, we did not earmark funds in the budget to say, we're going to have that kind of assistance" (Informant 21, Small, Disease-Focused Program)

"The clinic actually has extensive social work resources. So, each of the patients already has a social worker. The families have a social worker assigned to them, as part of their cancer care. So, we didn't complement them.” (Informant 02, Small Disease-Focused Program)

"Because we're partnering with a health care system, and these are patients within the system, the system has ways in which they help with transportation, right, for patients to get to and from and so forth." (Informant 25, Medium Disease-Agnostic Program)

RoR, return of results.

ing the results however they would normally do it if they had ordered the clinical exome." (Informant 28, Small Disease-Focused Program).

Informants also shared their experiences collaborating with clinicians to best serve the needs of research participants. Taking time to both build relationships with clinicians and educate them about genomics were mentioned as important components to improve the RoR process.

\section{Research and Clinical Team Responsibilities}

Program informants differed regarding whether it was the research team's obligation to directly return results to participants (shown in Table 2). Thirty-seven percent of programs used research funding to support genetic counselors, medical geneticists, or clinicians to return results. "It gets very close to the level between research and clinical. Part of the research program is to have a genetic counselor give the results (who) therefore will be making the referrals to get things done." (Informant 10, Small Disease-Agnostic Program).

Genomic programs reported that responsibility for certain ancillary support services for LRRP was assigned to either the clinical or research team. Few genomic programs assumed responsibility for coordinating follow-up medical care for participants. Fifteen genomic programs provided an RoR session but did not provide referrals for follow-up or specialty care for participants. "But as a research study, we can't really take on the responsibility of then, like, making appointments or making sure they've got insurance to get access to it or anything. We've got to just leave that in their hands." (Informant 17, Medium Disease-Focused Program).

Informants were asked about other support services offered to participants including insurance enrollment, financial assistance, referrals to social work, transportation assistance, and language interpretation services."If 
Table 3. Barriers for LRRP following RoR

\begin{tabular}{ll}
\hline Themes & Quotes \\
\hline $\begin{array}{l}\text { Financial } \\
\text { Challenges }\end{array}$ & $\begin{array}{l}\text { "With respect to, getting access to paying for that appointment or access to, like, different screening } \\
\text { programs. That's beyond me (the study) can't pay for anything beyond, the study-related activities. None of } \\
\text { the downstream care. Which is tough, I know." (Informant 31, Small Disease-Focused Program) }\end{array}$ \\
\cline { 2 - 2 } & "We try to use the available resources through the health system to cover care when possible. It certainly is a \\
& burden for some patients to receive a result." (Informant 18, Large Disease-Agnostic Program)
\end{tabular}

"Like, number of times we contact; number of times we outreach. Number of times is just significantly more in the underserved compared to the (general population) - just small things like that, that take a lot more resources to contact underserved populations than not.” (Informant 01, Small Disease-Agnostic Program)

"Again, because we recruit within the institution with people who do get health care here, I would expect that it wouldn't be as much of a hardship for them to come back here and that they have services set up so that they have transportation to get to the institution here." (Informant 26, Small, Disease-Agnostic Program)

Participant Education and Genomic Literacy
"The biggest worry we have is that if they have an uninformative result, we don't find any variants, they might interpret that to mean that they are not at risk for (anything)." (Informant 13, Small Disease-Agnostic Program)

"I would say, it gets quite uncomfortable because as the language becomes more simplified, it also becomes less precise. And so, for those of us who understand all the technical terms, it actually feels like you're not saying - making a correct statement because it's so simplified. So, I think that that's why it's really hard to make it simple, but also make it be an accurate statement" (Informant 23, Small Disease-Focused Program)

"The word database reads at a pretty high reading level. Unpacking the concept of a biobank reads at a pretty high reading level. And so, no matter what you do with sentences around the sentence that introduces this concept of a biobank or a database, still, you're using that word - a compound, complex word" (Informant 07, Medium Disease-Agnostic Program)

"I think there is an over emphasis on genetic literacy. I think that people use all kinds of analogies, care repairs, whatever. People need to - people need to have enough knowledge to make an informed decision, that doesn't mean they have to understand how it all works. In my experience doing clinical genetics for 25 years is that we spend too much time trying to teach people Genetics 101. From our interviews from parents, our discussions with parents, parents basically want to know, 'What's important to me and my child?'” (Informant 24, Small Disease-Focused Program)

LRRP, low-resource research participants; RoR, return of results.

somebody shows up and they don't have coverage, the social worker is going to help them apply through the health connector to get coverage. But it's not done through our study per se, it's done through the hospital." (Informant 17, Medium Disease-Agnostic Program).

Many program informants recognized a need for their research participants to access these ancillary and social care services but did not express a responsibility to provide them, instead attributing responsibility to the affiliated health-care system or clinical team.

\section{Barriers for LRRP following RoR}

Financial Challenges

LRRP may experience significant downstream barriers to accessing medical care after the RoR (shown in Table 3). Many genomic program informants and their IRBs were aware of this potential concern and tried to approach it in different ways. One investigator characterized an exchange between their research team and the IRB. "They were like, 'We can't offer this test to uninsured people because they can't get the downstream care,' and 
the IRB said, 'No, that's not ethical, you need to, you can't just deny people access to federally funded research." (Informant 31, Small Disease-Focused Program).

However, while many programs were aware that LRRP may not have the resources to act on the results returned, they felt ill-equipped to meet this challenge."But, yeah, it's really the downstream. Once you deliver a result, and there's a change in medical management, at least trying to facilitate participants getting the care they need is important, and I think just figuring that out is challenging." (Informant 25, Medium Disease-Agnostic Program).

LRRP also face difficulties accessing routine genomic testing through standard medical care due to their uninsured or underinsured statuses (shown in Table 3). Investigators expressed discomfort that some LRRP may be enrolling in their research programs to access genomic tests that they might not otherwise receive."One of the concerns we've had, vis-à-vis return of results when we're doing a population screen like this is that people who have medical indications for testing would use this testing as a substitute for getting a formal clinical evaluation." (Informant 13, Small Disease-Agnostic Program).

Some investigators were less concerned about this and more willing to have their research serve as a means for participants to access genomic testing. "If that's the one way that they can get a test covered, even if it's a sub-optimal test, then there's of course no reason to steer anybody away from that." (Informant 18, Large Disease-Agnostic Program).

\section{Limited Investigator Resources for Ancillary Care}

When working with LRRP, many researchers experienced an increased demand for both financial and time resources. Programs that only recruited existing patients from within their health-care system were less likely to report an increased demand on their time for participant scheduling and transportation. It was commonly stated, however, that LRRP required more time and attention throughout every research phase, from recruitment to follow-up after the RoR session."I would suggest that everything takes a huge amount of more time. You have to be increasingly attentive. You have to be very careful about being respectful. You have to work hard on your materials. You have to reach out to the hospitals where you're recruiting so that they're comfortable." (Informant 12, Small Disease-Agnostic Program).

\section{Participant Education and Genomic Literacy}

Awareness of participant literacy is an important aspect of social care, and informants were divided on how they accommodated varying levels of genomic literacy, as well as the value of genomic literacy testing of participants (shown in Table 3). Four programs consulted literacy experts when developing their materials. Most programs simply complied with their institutional IRB guidelines, which were typically between 6 th and 8 th grade reading level.

\section{Informants' Attitudes toward RoR and Ancillary \\ Medical and Social Care for LRRP \\ Providing Medical and Social Care}

Due to the additional support required for LRRP in the research programs, some informants questioned the value of returning genomic research results to LRRP who may have more pressing needs."They need so much else, that's potentially more important, like food security and access to stay in the country. I think genetics is important, but I really don't think it's more important than a lot of things. Maybe just being one step away from people who don't even have access to a primary doctor to check their diabetes. Take it one step at a time." (Informant 31, Small Disease-Focused Program).

Other researchers felt strongly about working with LRRP and explained the invaluable nature of genomic information for all populations regardless of their circumstances. Another informant shared:"But I also am just very careful about saying somehow that, "Oh, this is like low-priority because there are so many bigger problems," because I remember once talking to a pediatric endocrinologist, and she was like, Yeah, I might have a couple patients who might have MODY (Maturity Onset Diabetes of Youth), but they have bigger problems. One of them is in the foster care system. And to me, I was like, "Well, that's too bad that that's the way you think of it. You could actually improve that child's quality of life." (Informant 16, Small Disease-Focused Program).

Informant attitudes were also influenced by their state health insurance marketplaces [33]. We sampled research programs nationwide and found informants in states with expanded Medicaid were less likely to report lack of health insurance as a barrier to genomic testing for LRRP.

Variants of Uncertain Significance Reclassification and Re-Contacting Study Participants

The same barriers that prevent LRRP from utilizing genomics results can disproportionately affect their ability to be recontacted and use the new genomic information if a change in the classification of a variant is made. Informants expressed a variety of perspectives on reanalyzing and recontacting participants based on possible re- 
Table 4. Solutions to meet LRRP community needs

\begin{tabular}{ll}
\hline Themes & Quotes \\
\hline Community & "We work closely with the community board, both the patient side and, also, on the physician side. And so, we work with them \\
Engagement & $\begin{array}{l}\text { - all the way through even from the point where we were designing this study and drawing a grant, to figure out how to do that } \\
\text { in a way that would be engaging to the participants we would like to recruit. We also work with a network of primary care } \\
\text { physicians and hear their perspectives of how we would do this. And then, we come up with strategies based on the experience } \\
\text { of researchers in our study who run several different programs. We come up with strategies for things like engaging people in } \\
\text { the study, retaining people in the study, and communicating effectively to participants in the study." (Informant 27, Small } \\
\text { Disease-Focused Program) }\end{array}$
\end{tabular}

"Recently we wanted to learn more about what types of really specific recommendations for follow-up we could give to patients. And we had very helpful feedback from our ethics council and our clinical advisory council on really how to make those type of recommendations to patients that aligns with their practices and their, kind of, approach." (Informant 18, Large DiseaseAgnostic Program)

Smartphone "Well, a lot of them were doing it on their smartphones. That's why, you know, it worked more in the beginning of the month when they had more minutes to be able to fill out the questionnaires. Most all of them had smartphones to be able to do that. They may not have had computers and computers at home, but at least on their smartphones they could." (Informant 01, Small Disease-Agnostic Program)

"So interestingly enough, with smartphones we've seen, you know, access-especially in terms of our underserved community is actually quite high. And so, we've designed everything so it can be done from your mobile phone. Doesn't have to be a computer or other things. And so, I think that's actually been another good equalizer in terms of participation." (Informant 17, Medium Disease-Focused Program)

Flexible

Scheduling

"People work 9:00 a.m. to 5:00 p.m., and some of the medically underserved work nights, or they may work evenings.

Traditional studies in academia are done, you know, from, like, 9:00 a.m. to 5:00 p.m., and we've adjusted hours and times and days to try to do some more at the beginning of the month." (Informant 01, Small Disease-Agnostic Program)

"I would say the other thing we did is many of the families in low resource settings could not come during the day because they had to work. And so, we would make the consent process available to them on nights and weekends." (Informant 02, Small Disease-Agnostic Program)

Biological Sample Collection

"We do have the option to draw blood if people want it. Everyone selects the saliva kit because it's easier. They don't have to come back into the hospital for a blood draw." (Informant 20, Small Disease-Agnostic Program)

"We looked into, like, how people could get their blood drawn remotely, but it's harder than it sounds. And so, we kind of turned to saliva, which the lab wasn't thrilled about, because saliva can be harder to work with, but, you know, I think we finally got to the point where it was like, "Okay, this will just make things so much easier." Because, really, all the communications can be done remotely, you know, all can be done through like, you know, telephone or video." (Informant 16, Small DiseaseFocused Program)

Patient Support Groups

"I know once they get genetic information, our brochures have whatever information is available about patient organization groups with that same genetic condition." (Informant 17, Medium Disease-Focused Program)

"We include support group information in the written materials that we send to everybody. And of course, for those patients who come to see us, that's a routine part of our genetic counseling." (Informant 18, Large Disease-Agnostic Program)

Insurance Enrollment Assistance

“The study doesn't, but the hospitals do. I think that's a big focus. If somebody shows up and they don't have coverage, they're going to get - the social worker is going to help them apply through the health connector to get coverage. It's not done through our study per se, it's done through the hospital.” (Informant 19, Medium Disease-Agnostic Program)

"If we've identified a patient, let's say, and they need, you know, more intense clinical management, screening, et cetera, and don't have insurance, we would likely refer them to the health care system who deals with it, because those are their patients. It's not that we won't be doing it; it's just the center here, in our capacity, that would probably be something that would be turned to the health care system." (Informant 25, Medium Disease-Agnostic Program)

Use of Charity or Philanthropic Funding to Support LRRP

"We'll ask for authorization for genetic testing. It'll be denied. Then we'll have, you know, some back and forth with us saying it's important and then the peer-to-peer discussions with insurance companies. And after that, you know, we basically give up and use our philanthropic funds." (Informant 30, Small Disease-Focused Program)

Referrals to Social Work Services
"We have a very active social work department for all of our patients. Because a lot of them have trouble with insurance. We're helping get insurance for their clinical care. And genetic services are included in that." (Informant 30, Small Disease-Focused Program)

"It would be embedded in the genetic counseling in some cases; and, if they needed further referral, it would be through the social services group at the institution." (Informant 13, Small Disease-Agnostic Program)

LRRP, low-resource research participants. 


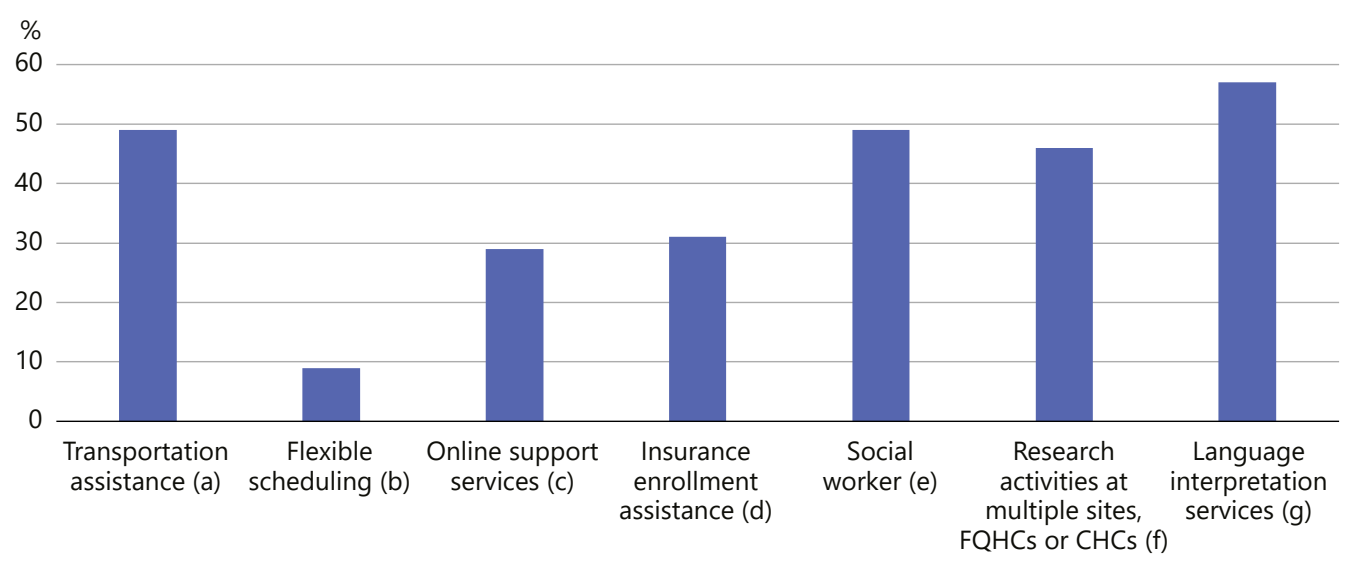

Fig. 1. Support services offered to research participants. a Assistance provided for participant to arrive at research appointments without a significant financial burden. b Research appointments outside of working hours ( 9 a.m. to 5 p.m.) to accommodate employed participants. c Programs provided "mobile-friendly" resources for participants without access to computers. d Programs provided enrollment assistance for research participants lacking health insurance. e Programs with a connection to a social worker

classification of variants of uncertain significance. Fiftyfour percent of programs were willing to provide periodic updates to participants. The nature of these updates ranged from newsletters with broad information on the laboratory activities to the reinterpretation of individual genomic results. Some informants believed that their research teams did not have adequate time to recontact participants. Others felt more inclined to share variants of uncertain significance updates with participants but recognized program resource constraints."I think the thing with re-contacting patients is everybody thinks it's a good idea, but then you have to kind of find a way that's actually feasible. So, we haven't made any promises about that, but we've left the possibility open and given people the chance to consent to continue to be contacted." (Informant 16, Small Disease-Focused Program).

\section{Solutions to Meet LRRP Community Needs}

In Table 4 and Figure 1, we report the strategies developed by some programs to support study participants. Although several programs highlighted actionable solutions that they found useful to better serve the LRRP community, informants were generally unable to define solutions to reduce the difficulties faced by LRRP in genomic studies. There were additional difficulties with recruitment whether within their own research program or through an academic institution or hospital. f To increase access to programs, research sites included Federally Qualified Health Centers and Community Health Centers that commonly serve LRRP. g Language interpretation and translation services were offered to nonEnglish-speaking participants. LRRP, low-resource research participants.

and inclusion of LRRP in programs, even when the LRRP were preexisting patients at the research program's academic institution or health-care system. Some programs provide specific social support services and ancillary care for their research participants, which could be of benefit for LRRP (shown in Figure 1). Overall, there was no consensus on how to deliver these services and no agreement that research programs had any obligation to provide these services to their participants, or LRRP in particular.

\section{Discussion}

Policies and practices regarding the return of genomic results to research participants are evolving. The NASEM report supports the return of medically actionable genomic research results and explains that the feasibility of return and the potential value for the participant increases the necessity to return genomic results. However, the NASEM report did not provide guidelines regarding the support participants may need after results are returned [2]. In general, returning results to participants of genomic studies has become more widely accepted; yet, there is still much debate regarding best practices. Further study of how to meet community needs of LRRP in ge- 
nomic research programs following RoR is needed to identify methods to ethically and equitably return genomic research results to members of these communities. Genomic research programs embed in low-resource settings such as federally qualified health centers have identified the need to consider social determinants of health in RoR processes, but there are no standard practices to achieve this goal [21]. This study describes a range of RoR structures in research programs, as well as the specific post-RoR support that researchers may offer to LRRP. Our findings show that informants recognized that some participants face greater barriers to act upon their genomic results, and some research programs lacked plans or resources to address the barriers that LRRP face.

To accomplish the scientific objective of diversifying research cohorts, it is important for researchers to establish partnerships with health professionals and healthcare systems that serve these populations such as safetynet hospitals or federally qualified health centers. However, barriers to genomic research participation can extend well beyond the recruitment stage. The COVID-19 pandemic has further highlighted and exposed the disparities in health care delivery and health outcomes that low-resource populations experience as well as the gaps in the USA's current fragmented health-care system [43]. As more LRRP begin to receive medically actionable research results, investigators should consider their ethical responsibility to provide appropriate ancillary care to their research participants.

Some informants stated that they made few accommodations for the LRRP in their programs. Others believed that providing additional accommodations for LRRP was outside the scope of the research program. Researchers who expressed a responsibility to support LRRP were often reliant on external resources and assistance, which limited the support they could provide.

Researchers must recognize the barriers that LRRP may face downstream after receiving medically actionable results. Ancillary care should be considered for all participants when returning genomic results, with recognition of LRRP's distinct circumstances. These ancillary care services include insurance enrollment assistance, flexible RoR session scheduling, transportation assistance, and referrals to social work services in order to better equip LRRP to address the downstream consequences of the results returned to them. These issues of follow-up care are complex, especially when the health of family members may be impacted as well. However, while the role of research programs providing ancillary care remains a debate, there are steps that programs can take to be inclusive of LRRP needs (Table 4;
Fig. 1). We are not prescribing what ancillary and social care must be provided. Instead, we contend that researchers have an obligation to incorporate within their study design an RoR plan that addresses the special needs of LRRP. This plan should be based upon available resources, the research environment, and the unique needs of study participants. We recognize that implementing the services informants determined to be important must be incorporated within the study budget. This is not an issue unique to genomics and precision medicine. Difficulties with downstream barriers to care is a major issue in clinical trials across many disciplines. The genomics community has an opportunity to provide leadership in developing approaches to ethically return results to all research participants in translational research.

Of concern, some informants believed that genomic sequencing and testing was not a priority for LRRP because of other more pressing issues in their lives, such as housing and food insecurity. This belief could negatively impact the participation of underserved communities in research and the RoR process and exacerbate health care disparities. The genomics community continues to debate whether the return of genomic research results is an ethical responsibility. Inclusion of all individuals in research studies is a scientific and moral mandate, and to enroll only people who can afford to act on their genomic results would be unjust. There is a need for the biomedical research community to further develop best practices to meet the needs of LRRP participating in genomics research. Genomics research has made great strides to be inclusive of a diverse set of participant populations and institutions, and RoR policies should take this variation into account.

\section{Limitations}

This study has several limitations. First, our sample of genomic research programs or consortia may not be representative of the viewpoints of all researchers involved in this area of research. Second, all semi-structured interviews were conducted by phone instead of in person. Inperson interviews allow interviewers and interviewees to utilize body language, facial expressions, and other nonverbal cues to provide additional context to the discussion that cannot be employed on the phone [44].

We also have not assessed the viewpoints of LRRP enrolled in genomic research programs and what support they feel is needed after results are returned. As research includes more diverse participants, it is important to examine what support services LRRP perceive to be helpful in making their participation in genomic research more beneficial to them. 


\section{Conclusion}

The nature and extent of genomic research programs' responsibilities toward supporting LRRP after results are returned are unclear. The findings of this paper suggest that the resource status of participants should be considered when creating policy surrounding returning genomic results to research participants in the USA. While this consideration may place researchers in the position of becoming involved in addressing systemic barriers of the US health-care system, it is necessary in order to minimize potential harm to research participants from failing to address actionable genomic results.

Tackling this issue is 2-fold. First, there is a need for empirical research examining the necessary infrastructure of support and ancillary care for LRRP in genomic studies. Researchers, study participants, and clinicians should collaborate to develop best practices and policies to properly support LRRP and similar communities throughout the RoR process and for any follow-up care. Second, IRBs and scientific guidance committees should consider the variability in structure of research programs and consortia conducting genomics research and adapt recommendations accordingly. Incorporating LRRP and other diverse research participants in genomic research is merely a necessary first step toward meeting scientific and ethical goals of enrolling diverse populations. It is also necessary to identify the barriers that affect these individuals and communities to ensure their equitable participation in genomic research. Future research should aim to assess the unique perspectives of LRRP to ensure that their viewpoints are incorporated prospectively in practices and policies. This will provide insight into the challenges of this population in genomic research and in research more broadly.

LRRP in genomic studies are key stakeholders in the RoR process. Identifying their needs and perspectives is imperative to improve the RoR process and, in turn, to achieve justice-based goals in providing equitable potential benefits from research involving genomic sequencing and RoR.

\section{Acknowledgements}

The authors like to thank the study informants from the research programs that participated in the study who generously provided their time. We would like to thank Adrian Lee and Lucia Hindorff for their assistance in piloting the interview guide. We would also like to thank Wylie Burke, Lucia Hindorff, Faith Fletcher, and Laura Koehly for their thoughtful feedback on early drafts of this article. The views expressed are the authors' own, they do not represent the views or policies of the National Human Genome Research Institute, National Institutes of Health, or the Department of Health and Human Services.

\section{Statement of Ethics}

All procedures performed in studies involving human participants were in accordance with the ethical standards of the Institutional and/or National Research Committee and with the 1964 Helsinki Declaration and its later amendments or comparable ethical standards. Subjects have given their informed consent, and the study protocol was approved by the Institutional Review Board at the National Human Genome Research Institute (14-HG-0125, clinicaltrials.gov: NCT02156102).

\section{Conflict of Interest Statement}

The authors have no conflicts of interest to declare.

\section{Funding Sources}

This work was supported in part by the Division of Intramural Research, National Human Genome Research Institute (NHGRI) ZIAHG200324-16 and ZIAHG200403-01.

\section{Author Contributions}

M.B.R. and V.L.B. were responsible for the concept of the work and research design. M.B.R. was responsible for data collection. M.B.R. and K.E.C. were responsible for data analysis, interpretation of data, and drafting of the manuscript. L.S.P. and V.L.B. made substantial contributions to interpretation of the data and revising the manuscript for important intellectual content. V.L.B. supervised data collection, interpretation of data, and drafting and revising of the manuscript. All authors discussed, drafted, and critically reviewed the manuscript and approved the final version.

\section{References}

1 Burke W, Evans BJ, Jarvik GP. Return of results: ethical and legal distinctions between research and clinical care. Am J Med Genet C Semin Med Genet. 2014 Mar;166C(1):10511.

2 National Academies of Sciences, Engineering, and medicine; health and medicine division; board on health sciences policy; committee on the return of individual-specific research results generated in research laboratories. Returning individual research results to participants: guidance for a new research paradigm. In: Downey AS, Busta ER, Mancher M, Botkin JR, editors. Washington (DC): National Academies Press (US); 2018 Jul 10. 
3 Dixon-Woods M, Tarrant C, Jackson CJ, Jones DR, Kenyon S. Providing the results of research to participants: a mixed-method study of the benefits and challenges of a consultative approach. Clin Trials. 2011 June; 8(3):330-41.

4 Ashida S, Koehly LM, Roberts JS, Chen CA, Hiraki S, Green RC. The role of disease perceptions and results sharing in psychological adaptation after genetic susceptibility testing: the reveal study. Eur J Hum Genet. 2010 Dec; 18(12):1296-301.

5 Dal-Ré R, Katsanis N, Katsanis S, Parker LS, Ayuso C. Managing incidental genomic findings in clinical trials: fulfillment of the principle of justice. PLoS Med. 2014 Jan;11(1): e1001584.

6 Horowitz CR, Orlando LA, Slavotinek AM, Peterson J, Angelo F, Biesecker B, et al. The genomic medicine integrative research framework: a conceptual framework for conducting genomic medicine research. Am J Hum Genet. 2019 June;104(6):1088-96.

7 Hindorff LA, Bonham VL, Ohno-Machado L. Enhancing diversity to reduce health information disparities and build an evidence base for genomic medicine. Per Med. 2018 Sep; 15(5):403-12.

8 Amendola LM, Berg JS, Horowitz CR, Angelo F, Bensen JT, Biesecker BB, et al. The clinical sequencing evidence-generating research consortium: integrating genomic sequencing in diverse and medically underserved populations. Am J Hum Genet. 2018 Sep;103(3): $319-27$.

9 Mathew SS, Barwell J, Khan N, Lynch E, Parker M, Qureshi N. Inclusion of diverse populations in genomic research and health services: genomix workshop report. J Community Genet. 2017 Oct;8(4):267-73.

10 Sankar PL, Parker LS. The precision medicine initiative's all of us research program: an agenda for research on its ethical, legal, and social issues. Genet Med. 2017 Jul;19(7):74350.

11 Horowitz CR, Sabin T, Ramos M, Richardson LD, Hauser D, Robinson M, et al. Successful recruitment and retention of diverse participants in a genomics clinical trial: a good invitation to a great party. Genet Med. 2019 Oct; 21(10):2364-70.

$12 \mathrm{Kraft}$ SA, Doerr M. Engaging populations underrepresented in research through novel approaches to consent. Am J Med Genet C Semin Med Genet. 2018 Mar;178(1):75-80.

13 Shaibi GQ, Kullo IJ, Singh DP, Sharp RR, De Filippis E, Cuellar I, et al. Developing a process for returning medically actionable genomic variants to latino patients in a federally qualified health center. Public Health Genomics. 2018;21(1-2):77-84.

14 Fossey R, Kochan D, Winkler E, Pacyna JE, Olson J, Thibodeau S, et al. Ethical considerations related to return of results from genomic medicine projects: the eMERGE network (phase III) experience. J Pers Med. 2018 Jan;8(1):2.
15 Beskow LM, O'Rourke PP. Return of genetic research results to participants and families: IRB perspectives and roles. J Law Med Ethics. 2015;43(3):502-13.

16 Groisman IJ, Mathieu G, Godard B. Use of next generation sequencing technologies in research and beyond: are participants with mental health disorders fully protected? BMC Med Ethics. 2012 Dec;13:36.

17 Chan B, Facio FM, Eidem H, Hull SC, Biesecker LG, Berkman BE. Genomic inheritances: disclosing individual research results from whole-exome sequencing to deceased participants' relatives. Am J Bioeth. 2012;12(10):18

18 Holm IA, Savage SK, Green RC, Juengst E, McGuire A, Kornetsky S, et al. Guidelines for return of research results from pediatric genomic studies: deliberations of the Boston children's hospital gene partnership informed cohort oversight board. Genet Med. 2014 Jul; 16(7):547-52.

19 McGuire AL, Joffe S, Koenig BA, Biesecker BB, McCullough LB, Blumenthal-Barby JS, et al. Point-counterpoint. Ethics and genomic incidental findings. Science. 2013 May; 340(6136):1047-8.

20 Wolf SM, Branum R, Koenig BA, Petersen GM, Berry SA, Beskow LM, et al. Returning a research participant's genomic results to relatives: analysis and recommendations. J Law Med Ethics. 2015;43(3):440-63.

21 Shaibi GQ, Kullo IJ, Singh DP, Hernandez V, Sharp RR, Cuellar I, et al. Returning genomic results in a federally qualified health center: the intersection of precision medicine and social determinants of health. Genet Med. 2020 Sep;22(9):1552-9.

22 Beauchamp TL, Childress JF. Principles of biomedical ethics. 7th ed. New York: Oxford University Press; 2012.

23 Beskow LM, Burke W. Offering individual genetic research results: context matters. Sci Transl Med. 2010 Jun 30;2(38):38cm20.

24 Darnell AJ, Austin H, Bluemke DA, Cannon RO, Fischbeck K, Gahl W, et al. A clinical service to support the return of secondary genomic findings in human research. Am J Hum Genet. 2016;98(3):435-41.

25 Dal-Re' R, Katsanis N, Katsanis S, Parker LS, Ayuso C. Managing incidental genomic findings in clinical trials: fulfillment of principle of justice. PLoS Med. 2014;11(1): e1001584.

26 Berkman BE, Hull SC, Eckstein L. The unintended implications of blurring the line between research and clinical care in a genomic age. Per Med. 2014;11(3):285-95.

27 Bombard Y, Brothers KB, Fitzgerald-Butt S, Garrison NA, Jamal L, James CA, et al. The responsibility to recontact research participants after reinterpretation of genetic and genomic research results. Am J Hum Genet. 2019;104:578-95.
28 Richardson HS, Belsky L. The ancillary-care responsibilities of medical researchers. An ethical framework for thinking about the clinical care that researchers owe their subjects. Hastings Cent Rep. 2004 Jan-Feb;34(1):2533.

29 Richardson HS. Moral engagements: the ancillary-care obligations of medical researcher: Oxford University Press; 2016 June. Vol. 30; p. $317-24$.

30 Richardson HS. Incidental findings and ancillary-care obligations. J Law Med Ethics. 2008; 36(2):256-11.

31 Richardson HS, Eyal N, Campbell JI, Haberer JE. When ancillary care clashes with study aims. N Engl J Med. 2017 Sep;377(13):1213-5.

32 National Academies of Sciences. Engineering, and Medicine. 2019. Integrating social care into the delivery of health care: Moving upstream to improve the nation's health. Washington: The National Academies Press; [cited 2020 Dec 5].

33 Millum J. Post-trial access to antiretrovirals: who owes what to whom? Bioethics. 2011; 25(3):145-54.

34 Haire BG, Ogundokun O. Ethics of ancillary care in clinical trials in low income countries: a Nigerian case study. Afr J Reprod Health. 2014;18(3):135-42.

35 Sullivan HK, Berkman BE. Incidental findings in low-resource settings. Hastings Cent Rep. 2018;48(3):20-8.

36 Hyder AA, Merritt MW. Ancillary care for public health research in developing countries. JAMA. 2009;302(4):429-31.

37 Cho HL, Danis M, Grady C. The ethics of uninsured participants accessing healthcare in biomedical research: a literature review. Clin Trials. 2018;15(5):509-21.

38 Jacobson N, Krupp A, Bowers BJ. Planning for ancillary care provision. J Empir Res Hum Res Ethics. 2016;11(2):129-34.

39 Veiraiah A. Responsibility for ancillary care in clinical trials: research patrimony and unintended coercion are hazards. BMJ. 2004; 329:232.

40 Dressler LG, Smolek S, Ponsaran R, Markey JM, Starks H, Gerson N, et al. IRB perspectives on the return of individual results from genomic research. Genet Med. 2012 Feb; 14(2):215-22.

41 Meacham MC, Starks H, Burke W, Edwards K. Researcher perspectives on disclosure of incidental findings in genetic research. J Empir Res Hum Res Ethics. 2010 Sep;5(3):31-41.

42 Hsieh HF, Shannon SE. Three approaches to qualitative content analysis. Qual Health Res. 2005 Nov; 15(9):1277-88.

43 Muñoz-Price LS, Nattinger AB, Rivera F, Hanson R, Gmehlin CG, Perez A, et al. Racial disparities in incidence and outcomes among patients with COVID-19. JAMA Netw Open. 2020 Sep 1;3(9):e2021892.

44 Block ES, Erskine L. Interviewing by telephone: specific considerations, opportunities, and challenges. Int J Qual Methods. 2012 Sep; 11(4):428-45. 